\title{
Note on the Persistent Property of a Discrete Lotka-Volterra Competitive System with Delays and Feedback Controls
}

\author{
Xiangzeng Kong, ${ }^{1,2}$ Liping Chen, ${ }^{1,2}$ and Wensheng Yang ${ }^{1,2}$ \\ ${ }^{1}$ Key Lab of Network Security and Cryptology, Fujian Normal University, Fuzhou 350007, China \\ ${ }^{2}$ School of Mathematics and Computer Science, Fujian Normal University, Fuzhou 350007, China \\ Correspondence should be addressed to Xiangzeng Kong, xzkong@fjnu.edu.cn
}

Received 26 June 2010; Accepted 12 September 2010

Academic Editor: P. J. Y. Wong

Copyright (C) 2010 Xiangzeng Kong et al. This is an open access article distributed under the Creative Commons Attribution License, which permits unrestricted use, distribution, and reproduction in any medium, provided the original work is properly cited.

A nonautonomous $N$-species discrete Lotka-Volterra competitive system with delays and feedback controls is considered in this work. Sufficient conditions on the coefficients are given to guarantee that all the species are permanent. It is shown that these conditions are weaker than those of Liao et al. 2008.

\section{Introduction}

Traditional Lotka-Volterra competitive systems have been extensively studied by many authors [1-7].The autonomous model can be expressed as follows:

$$
u_{i}^{\prime}(t)=b_{i} u_{i}(t)\left[1-\sum_{j=1}^{N} a_{i j} u_{j}(t)\right], \quad i=1, \ldots, N
$$

where $b_{i}>0, a_{i i}>0, a_{i j} \geq 0(i \neq j), u_{i}(t)$ denoting the density of the $i$ th species at time $t$. Montes de Oca and Zeeman [6] investigated the general nonautonomous $N$-species Lotka-Volterra competitive system

$$
u_{i}^{\prime}(t)=u_{i}(t)\left[b_{i}(t)-\sum_{j=1}^{N} c_{i j}(t) u_{j}(t)\right], \quad c_{i j} \geq 0, i=1, \ldots, N
$$


and obtained that if the coefficients are continuous and bounded above and below by positive constants, and if for each $i=2, \ldots, N$, there exists an integer $k_{i}<i$ such that

$$
\frac{\bar{b}_{i}}{\underline{c}_{i j}}<\frac{\underline{b}_{k i}}{\overline{\bar{c}}_{k_{i} j}}, \quad j=1, \ldots, i,
$$

then $u_{i} \rightarrow 0$ exponentially for $2 \leq i \leq N$, and $u_{i}(t) \rightarrow X^{*}$, where $X^{*}$ is a certain solution of a logistic equation. Teng [8] and Ahmad and Stamova [9] also studied the coexistence on a nonautonomous Lotka-Volterra competitive system. They obtained the necessary or sufficient conditions for the permanence and the extinction. For more works relevant to system (1.1), one could refer to [1-9] and the references cited therein.

However, to the best of the authors' knowledge, to this day, still less scholars consider the general nonautonomous discrete Lotka-Volterra competitive system with delays and feedback controls. Recently, in [1] Liao et al. considered the following general nonautonomous discrete Lotka-Volterra competitive system with delays and feedback controls:

$$
\begin{gathered}
x_{i}(n+1)=x_{i}(n) \exp \left\{b_{i}(n)-\sum_{j=1}^{N} a_{i j}(n) x_{j}\left(n-\tau_{i j}\right)-d_{i}(n) u_{i}(n)\right\}, \\
\Delta u_{i}(n)=r_{i}(n)-e_{i}(n) u_{i}(n)+c_{i}(n) x_{i}\left(n-\sigma_{i}\right), \quad i=1,2, \ldots, N, \\
x_{i}(\theta)=\phi_{i}(\theta) \geq 0, \quad \theta \in \mathbb{N}[-\tau, 0]:=\{-\tau,-\tau+1, \ldots,-1,0\},
\end{gathered}
$$

where $x_{i}(n)(i=1,2, \ldots, N)$ is the density of competitive species; $u_{i}(n)$ is the control variable; $e_{i}(n): \mathbb{Z} \rightarrow(0,1)$; bounded sequences $r_{i}(n), c_{i}(n), b_{i}(n), a_{i j}(n)$, and $d_{i}(n): \mathbb{Z} \rightarrow \mathbb{R}^{+} ; \tau_{i j}$ and $\sigma_{i}$ are positive integer; $\mathbb{Z}, \mathbb{R}^{+}$denote the sets of all integers and all positive real numbers, respectively; $\Delta$ is the first-order forward difference operator $\Delta u_{i}(n)=u_{i}(n+1)-u_{i}(n) ; \tau=$ $\max \left\{\max _{1 \leq i, j \leq N} \tau_{i j}, \max _{1 \leq i \leq N} \sigma_{i}\right\}>0$.

In [1], Liao et al. obtained sufficient conditions for permanence of the system (1.4).

They obtained what follows.

Lemma 1.1. Assume that

$$
\min _{1 \leq i \leq N} M_{i} \Delta_{i}>1
$$

hold, then system (1.4) is permanent, where

$$
\begin{gathered}
M_{i} \Delta_{i}=\frac{\exp \left\{b_{i}^{u}-1\right\}}{a_{i i}^{l} \exp \left\{-b_{i}^{u} \tau_{i i}\right\}} \cdot \frac{a_{i i}^{u} \exp \left\{\tau_{i i}\left(\sum_{j=1}^{N} a_{i j}^{u} M_{j}+W_{i} d_{i}^{u}-b_{i}^{l}\right)\right\}}{b_{i}^{l}-\sum_{j=1, j \neq i}^{N} a_{i j}^{u} M_{j}-d_{i}^{u} W_{i}}, \\
W_{i}=\frac{r_{i}^{u}+c_{i}^{u} M_{i}}{e_{i}^{l}}, \quad M_{i}=\frac{\exp \left(b_{i}^{u}-1\right)}{a_{i i}^{l} \exp \left(-b_{i}^{u} \tau_{i i}\right)} .
\end{gathered}
$$


Since

$$
\exp \left\{b_{i}^{u}-1\right\}>0, \quad a_{i i}^{l} \exp \left\{-b_{i}^{u} \tau_{i i}\right\}>0, \quad a_{i i}^{u} \exp \left\{\tau_{i i}\left(\sum_{j=1}^{N} a_{i j}^{u} M_{j}+W_{i} d_{i}^{u}-b_{i}^{l}\right)\right\}>0
$$

Hence, the above inequality (1.5) implies

$$
b_{i}^{l}-\sum_{j=1, j \neq i}^{N} a_{i j}^{u} M_{j}-d_{i}^{u} W_{i}>0
$$

That is

$$
\begin{aligned}
b_{i}^{l} & >\sum_{j=1, j \neq i}^{N} a_{i j}^{u} M_{j}+d_{i}^{u} W_{i} \\
& =\sum_{j=1, j \neq i}^{N} a_{i j}^{u} M_{j}+d_{i}^{u} \frac{r_{i}^{u}+c_{i}^{u} M_{i}}{e_{i}^{l}} \\
& =\sum_{j=1, j \neq i}^{N} a_{i j}^{u} M_{j}+\frac{d_{i}^{u} r_{i}^{u}}{e_{i}^{l}}+\frac{d_{i}^{u} c_{i}^{u} M_{i}}{e_{i}^{l}} .
\end{aligned}
$$

It was shown that in [1] Liao et al. considered system (1.4) where all coefficients $r_{i}(n), c_{i}(n), d_{i}(n)$, $a_{i j}(n), e_{i}(n)$, and $b_{i}(n)$ were assumed to satisfy conditions (1.9).

In this work, we shall study system (1.4) and get the same results as [1] do under the weaker assumption that

$$
b_{i}^{l}>\sum_{j=1, j \neq i}^{N} a_{i j}^{u} M_{j}+\frac{d_{i}^{u} r_{i}^{u}}{e_{i}^{l}}
$$

Our main results are the following Theorem 1.2.

Theorem 1.2. Assume that (1.10) holds, then system (1.4) is permanent.

Remark 1.3. The inequality (1.9) implies (1.10), but not conversely, for

$$
\sum_{j=1, j \neq i}^{N} a_{i j}^{u} M_{j}+\frac{d_{i}^{u} r_{i}^{u}}{e_{i}^{l}} \leq \sum_{j=1, j \neq i}^{N} a_{i j}^{u} M_{j}+\frac{d_{i}^{u} r_{i}^{u}}{e_{i}^{l}}+\frac{d_{i}^{u} c_{i}^{u} M_{i}}{e_{i}^{l}}
$$

Therefore, we have improved the permanence conditions of [1] for system (1.4).

Theorem 1.2 will be proved in Section 2. In Section 3, an example will be given to illustrate that (1.10) does not imply (1.9); that is, the condition (1.10) is better than (1.9). 


\section{Proof of Theorem 1.2}

The following lemma can be found in [10].

Lemma 2.1. Assume that $A>0$ and $y(0)>0$, and further suppose that (1)

$$
y(n+1) \leq A y(n)+B(n), \quad n=1,2, \ldots
$$

Then for any integer $k \leq n$,

$$
y(n) \leq A^{k} y(n-k)+\sum_{i=0}^{k-1} A^{i} B(n-i-1)
$$

Especially, if $A<1$ and $B$ is bounded above with respect to $M$, then

$$
\lim _{n \rightarrow \infty} \sup y(n) \leq \frac{M}{1-A}
$$

(2)

$$
y(n+1) \geq A y(n)+B(n), \quad n=1,2, \ldots
$$

Then for any integer $k \leq n$,

$$
y(n) \geq A^{k} y(n-k)+\sum_{i=0}^{k-1} A^{i} B(n-i-1) .
$$

Especially, if $A<1$ and $B$ is bounded below with respect to $m^{*}$, then

$$
\lim _{n \rightarrow \infty} \inf y(n) \geq \frac{m^{*}}{1-A}
$$

Following comparison theorem of difference equation is Theorem 2.1 of [11, page 241].

Lemma 2.2. Let $n \in N_{n_{0}}^{+}=\left\{n_{0}, n_{0}+1, \ldots, n_{0}+l, \ldots\right\}, r \geq 0$. For any fixed $n, g(n, r)$ is a nondecreasing function with respect to $r$, and for $n \geq n_{0}$, following inequalities hold: $y(n+1) \leq$ $g(n, y(n)), u(n+1) \geq g(n, u(n))$. If $g\left(n_{0}\right) \leq u\left(n_{0}\right)$, then $y(n) \leq u(n)$ for all $n \geq n_{0}$.

Now let us consider the following single species discrete model:

$$
N(n+1)=N(n) \exp \{a(n)-b(n) N(n)\},
$$

where $\{a(n)\}$ and $\{b(n)\}$ are strictly positive sequences of real numbers defined for $n \in N=$ $\{0,1,2, \ldots\}$ and $0<a^{l} \leq a^{u}, 0<b^{l} \leq b^{u}$. Similarly to the proof of Propositions 1 and 3 in [12], we can obtain the following. 
Advances in Difference Equations

Lemma 2.3. Any solution of system (2.7) with initial condition $N(0)>0$ satisfies

$$
m \leq \lim _{n \rightarrow \infty} \inf N(n) \leq \lim _{n \rightarrow \infty} \sup N(n) \leq M,
$$

where

$$
M=\frac{1}{b^{l}} \exp \left\{a^{u}-1\right\}, \quad m=\frac{a^{l}}{b^{u}} \exp \left\{a^{l}-b^{u} M\right\} .
$$

The following lemma is direct conclusion of [1].

Lemma 2.4. Let $x(n)=\left(x_{1}(n), x_{2}(n), \ldots, x_{N}(n), u_{1}(n), u_{2}(n), \ldots, u_{N}(n)\right)$ denote any positive solution of system (1.4). Then there exist positive constants $M_{i}, W_{i}(i=1,2, \ldots, N)$ such that

$$
\lim _{n \rightarrow \infty} \sup x_{i}(n) \leq M_{i}, \quad \lim _{n \rightarrow \infty} \sup u_{i}(n) \leq W_{i}, \quad i=1,2, \ldots, N,
$$

where

$$
M_{i}=\frac{\exp \left(b_{i}^{u}-1\right)}{a_{i i}^{l} \exp \left(-b_{i}^{u} \tau_{i i}\right)}, \quad W_{i}=\frac{r_{i}^{u}+c_{i}^{u} M_{i}}{e_{i}^{l}} \quad(i=1,2, \ldots, N) .
$$

Proposition 2.5. Suppose assumption (1.10) holds, then there exist positive constant $m_{i}$ and $w_{i}$ such that

$$
\lim _{n \rightarrow \infty} \inf x_{i}(n) \geq m_{i}, \quad \lim _{n \rightarrow \infty} \inf u_{i}(n) \geq w_{i}
$$

Proof. We first prove $\lim _{n \rightarrow \infty} \inf x_{i}(n) \geq m_{i}$.

By Lemma 2.4 and by the first equation of system (1.4), we have

$$
\begin{aligned}
x_{i}(n+1) & =x_{i}(n) \exp \left\{b_{i}(n)-\sum_{j=1}^{N} a_{i j}(n) x_{j}\left(n-\tau_{i j}\right)-d_{i}(n) u_{i}(n)\right\} \\
& \geq x_{i}(n) \exp \left\{b_{i}(n)-\sum_{j=1}^{N} a_{i j}\left(M_{j}+\varepsilon\right)-d_{i}(n)\left(W_{i}+\varepsilon\right)\right\}
\end{aligned}
$$

for $n$ sufficiently large, then

$$
\prod_{s=n-\tau_{i i}}^{n-1} \frac{x_{i}(s+1)}{x_{i}(s)} \geq \exp \left\{\sum_{s=n-\tau_{i i}}^{n-1}\left(b_{i}(s)-\sum_{j=1}^{N} a_{i j}(s)\left(M_{j}+\varepsilon\right)-d_{i}(s)\left(W_{i}+\varepsilon\right)\right)\right\}
$$


Thus

$$
x_{i}\left(n-\tau_{i i}\right) \leq x_{i}(n) \exp \left\{\sum_{s=n-\tau_{i i}}^{n-1} D_{i}(s)\right\}
$$

where

$$
D_{i}(s)=\sum_{j=1}^{N} a_{i j}(s)\left(M_{j}+\varepsilon\right)+d_{i}(s)\left(W_{i}+\varepsilon\right)-b_{i}(s)
$$

From the second equation of system (1.4), we have

$$
\begin{aligned}
u_{i}(n) & =\left(1-e_{i}(n)\right) u_{i}(n)+c_{i}(n) x_{i}\left(n-\sigma_{i}\right)+r_{i}(n) \\
& \leq\left(1-e_{i}^{l}\right) u_{i}(n)+c_{i}(n) x_{i}\left(n-\sigma_{i}\right)+r_{i}(n) \\
& :=A_{i} u_{i}(n)+B_{i}(n) .
\end{aligned}
$$

Then, Lemma 2.1 implies that for any $k \leq n-\tau_{i i}$,

$$
\begin{aligned}
u_{i}(n) & \leq A_{i}^{k} u_{i}(n-k)+\sum_{j=0}^{k-1} A_{i}^{j} B_{i}(n-j-1) \\
& =A_{i}^{k} u_{i}(n-k)+\sum_{j=0}^{k-1} A_{i}^{j}\left[r_{i}(n-j-1)+c_{i}(n-j-1) x_{i}\left(n-j-1-\sigma_{i}\right)\right] \\
& \leq A_{i}^{k} u_{i}(n-k)+\sum_{j=0}^{k-1} A_{i}^{j}\left[r_{i}(n-j-1)+c_{i}^{u} \exp \left\{\left(j+1+\sigma_{i}\right) D_{i}^{u}\right\} x_{i}(n)\right] \\
& \leq A_{i}^{k} u_{i}(n-k)+\sum_{j=0}^{k-1} A_{i}^{j} r_{i}^{u}+\sum_{j=0}^{k-1} A_{i}^{j} c_{i}^{u} c_{i}^{u} \exp \left\{\left(j+1+\sigma_{i}\right) D_{i}^{u}\right\} x_{i}(n) \\
& \leq A_{i}^{k} W_{i}+\frac{1-A_{i}^{k}}{1-A_{i}} r_{i}^{u}+H_{i} x_{i}(n),
\end{aligned}
$$

where

$$
H_{i}=\left[\sum_{j=0}^{k-1} A_{i}^{j} c_{i}^{u} c_{i}^{u} \exp \left\{\left(j+1+\sigma_{i}\right) D_{i}^{u}\right\}\right]^{u}
$$

For any small positive constant $\varepsilon>0$, there exists a $K>0$ such that

$$
\left(d_{i}^{u} W_{i}-\frac{r_{i}^{u} d_{i}^{u}}{1-A_{i}}\right) A_{i}^{k}<\varepsilon \quad \forall k>K
$$


From the first equation of system (1.4), (2.18), and (2.20), we have

$$
\begin{aligned}
& x_{i}(n+1) \\
& \geq x_{i}(n) \exp \left\{b_{i}(n)-\sum_{j=1, j \neq i}^{N} a_{i j}(n) M_{j}-a_{i i}^{u} \exp \left\{\tau_{i i} D_{i}^{u}\right\} x_{i}(n)\right. \\
& \left.-d_{i}^{u} W_{i} A_{i}^{k}-\frac{1-A_{i}^{k}}{1-A_{i}} r_{i}^{u} d_{i}^{u}-d_{i}^{u} H_{i} x_{i}(n)\right\} \\
& =x_{i}(n) \exp \left\{b_{i}(n)-\sum_{j=1, j \neq i}^{N} a_{i j}(n) M_{j}-\frac{r_{i}^{u} d_{i}^{u}}{1-A_{i}}-\left(d_{i}^{u} W_{i}-\frac{r_{i}^{u} d_{i}^{u}}{1-A_{i}}\right) A_{i}^{k}\right. \\
& \left.-\left(a_{i i}^{u} \exp \left\{\tau_{i i} D_{i}^{u}\right\}+d_{i}^{u} H_{i}\right) x_{i}(n)\right\} \\
& \geq x_{i}(n) \exp \left\{b_{i}(n)-\sum_{j=1, j \neq i}^{N} a_{i j}(n) M_{j}-\frac{r_{i}^{u} d_{i}^{u}}{1-A_{i}}-\varepsilon-\left(a_{i i}^{u} \exp \left\{\tau_{i i} D_{i}^{u}\right\}+d_{i}^{u} H_{i}\right) x_{i}(n)\right\} .
\end{aligned}
$$

By Lemmas 2.2 and 2.3, we have

$$
\begin{aligned}
\lim _{n \rightarrow \infty} \inf x_{i}(n) \geq & \frac{b_{i}^{l}-\sum_{j=1, j \neq i}^{N} a_{i j}^{u} M_{j}-\left(r_{i}^{u} d_{i}^{u} / e_{i}^{l}\right)-\varepsilon}{a_{i i}^{u} \exp \left\{\tau_{i i} D_{i}^{u}\right\}+d_{i}^{u} H_{i}} \\
& \cdot \exp \left\{b_{i}^{l}-\sum_{j=1, j \neq i}^{N} a_{i j}^{u} M_{j}-\frac{r_{i}^{u} d_{i}^{u}}{e_{i}^{l}}-\varepsilon-\left(a_{i i}^{u} \exp \left\{\tau_{i i} D_{i}^{u}\right\}+d_{i}^{u} H_{i}\right) M_{i}\right\} .
\end{aligned}
$$

Setting $\varepsilon \rightarrow 0$ in (2.22) leads to

$$
\begin{aligned}
\lim _{n \rightarrow \infty} \inf x_{i}(n) \geq & \frac{b_{i}^{l}-\sum_{j=1, j \neq i}^{N} a_{i j}^{u} M_{j}-\left(r_{i}^{u} d_{i}^{u} / e_{i}^{l}\right)}{a_{i i}^{u} \exp \left\{\tau_{i i} D_{i}^{u}\right\}+d_{i}^{u} H_{i}} \\
& \cdot \exp \left\{b_{i}^{l}-\sum_{j=1, j \neq i}^{N} a_{i j}^{u} M_{j}-\frac{r_{i}^{u} d_{i}^{u}}{e_{i}^{l}}-\left(a_{i i}^{u} \exp \left\{\tau_{i i} D_{i}^{u}\right\}+d_{i}^{u} H_{i}\right) M_{i}\right\} .
\end{aligned}
$$

Thus,

$$
\lim _{n \rightarrow \infty} \inf x_{i}(n) \geq m_{i}
$$


where

$$
\begin{aligned}
m_{i}= & \frac{b_{i}^{l}-\sum_{j=1, j \neq i}^{N} a_{i j}^{u} M_{j}-\left(r_{i}^{u} d_{i}^{u} / e_{i}^{l}\right)}{a_{i i}^{u} \exp \left\{\tau_{i i} D_{i}^{u}\right\}+d_{i}^{u} H_{i}} \\
& \cdot \exp \left\{b_{i}^{l}-\sum_{j=1, j \neq i}^{N} a_{i j}^{u} M_{j}-\frac{r_{i}^{u} d_{i}^{u}}{e_{i}^{l}}-\left(a_{i i}^{u} \exp \left\{\tau_{i i} D_{i}^{u}\right\}+d_{i}^{u} H_{i}\right) M_{i}\right\} .
\end{aligned}
$$

Second, we prove $\lim _{n \rightarrow \infty} \inf u_{i}(n) \geq w_{i}$. For enough small $\varepsilon>0$, from the second equation of system (1.4), we have

$$
u_{i}(n+1)=\left(1-e_{i}(n)\right) u_{i}(n)+r_{i}(n)+c_{i}(n) x_{i}\left(n-\sigma_{i}\right) \geq r_{i}^{l}+c_{i}^{l}\left(m_{i}-\varepsilon\right)+\left(1-e_{i}^{u}\right) u_{i}(n)
$$

for sufficient large $n$. Hence

$$
u_{i}(n) \geq\left(1-e_{i}^{u}\right)^{n} u_{i}(0)+\frac{1-\left(1-e_{i}^{u}\right)}{e_{i}^{u}}\left(r_{i}^{l}+c_{i}^{l}\left(m_{i}-\varepsilon\right)\right)
$$

Thus, we obtain

$$
\lim _{n \rightarrow \infty} \inf u_{i}(n) \geq w_{i}
$$

This completes the proof.

\section{An Example}

In this section, we give an example to illustrate that (1.10) does not imply (1.9). Consider the two-species system with delays and feedback controls for $t \in(-\infty,+\infty)$

$$
\begin{aligned}
x_{1}(n+1) & =x_{1}(n) \exp \left\{\frac{1}{2}-2 x_{1}(n-1)-\frac{1}{2} x_{2}(n-3)-\frac{1}{2} u_{1}(n)\right\}, \\
x_{2}(n+1) & =x_{2}(n) \exp \left\{\frac{1}{2}-\frac{1}{2} x_{1}(n-3)-2 x_{2}(n-1)-\frac{1}{2} u_{2}(n)\right\}, \\
\Delta u_{1}(n+1) & =\frac{1}{8}-\frac{1}{2} u_{1}(n)+x_{1}(n-4), \\
\Delta u_{2}(n+1) & =\frac{1}{8}-\frac{1}{2} u_{2}(n)+x_{2}(n-8) .
\end{aligned}
$$

We have

$$
b_{1}^{l}=b_{2}^{l}=\frac{1}{2}, \quad M_{1}=M_{2}=\frac{1}{2}, \quad a_{12}^{u} M_{2}+d_{1}^{u} \frac{r_{1}^{u}}{e_{1}^{l}}=\frac{3}{8}, \quad a_{21}^{u} M_{1}+d_{2}^{u} \frac{r_{2}^{u}}{e_{2}^{l}}=\frac{3}{8} .
$$


So

$$
b_{1}^{l}>a_{12}^{u} M_{2}+d_{1}^{u} \frac{r_{1}^{u}}{e_{1}^{l}}, \quad b_{2}^{l}>a_{21}^{u} M_{1}+d_{2}^{u} \frac{r_{2}^{u}}{e_{2}^{l}}
$$

Therefore (1.10) holds.

But

$$
\frac{1}{2}=b_{1}^{l}<a_{12}^{u} M_{2}+d_{1}^{u} \frac{r_{1}^{u}+c_{1}^{u} M_{1}}{e_{1}^{l}}=\frac{7}{8}, \quad \frac{1}{2}=b_{2}^{l}<a_{21}^{u} M_{1}+d_{2}^{u} \frac{r_{2}^{u}+c_{2}^{u} M_{2}}{e_{2}^{l}}=\frac{7}{8} .
$$

Thus (1.9) does not hold.

\section{References}

[1] X. Liao, Z. Ouyang, and S. Zhou, "Permanence of species in nonautonomous discrete LotkaVolterra competitive system with delays and feedback controls," Journal of Computational and Applied Mathematics, vol. 211, no. 1, pp. 1-10, 2008.

[2] S. Ahmad, "On the nonautonomous Volterra-Lotka competition equations," Proceedings of the American Mathematical Society, vol. 117, no. 1, pp. 199-204, 1993.

[3] S. Ahmad and A. C. Lazer, "On the nonautonomous N-competing species problems," Applicable Analysis, vol. 57, no. 3-4, pp. 309-323, 1995.

[4] K. Gopalsamy, Stability and Oscillations in Delay Differential Equations of Population Dynamics, vol. 74 of Mathematics and Its Applications, Kluwer Academic Publishers, Dordrecht, The Netherlands, 1992.

[5] M. Kaykobad, "Positive solutions of positive linear systems," Linear Algebra and Its Applications, vol. 64, pp. 133-140, 1985.

[6] F. Montes de Oca and M. L. Zeeman, "Extinction in nonautonomous competitive Lotka-Volterra systems," Proceedings of the American Mathematical Society, vol. 124, no. 12, pp. 3677-3687, 1996.

[7] M. L. Zeeman, "Extinction in competitive Lotka-Volterra systems," Proceedings of the American Mathematical Society, vol. 123, no. 1, pp. 87-96, 1995.

[8] Z. D. Teng, "Permanence and extinction in nonautonomous Lotka-Volterra competitive systems with delays," Acta Mathematica Sinica, vol. 44, no. 2, pp. 293-306, 2001.

[9] S. Ahmad and I. M. Stamova, "Almost necessary and sufficient conditions for survival of species," Nonlinear Analysis. Real World Applications, vol. 5, no. 1, pp. 219-229, 2004.

[10] Y.-H. Fan and L.-L. Wang, "Permanence for a discrete model with feedback control and delay," Discrete Dynamics in Nature and Society, vol. 2008, Article ID 945109, 8 pages, 2008.

[11] L. Wang and M. Q. Wang, Ordinary Difference Equation, Xinjiang University Press, 1991.

[12] F. Chen, "Permanence and global attractivity of a discrete multispecies Lotka-Volterra competition predator-prey systems," Applied Mathematics and Computation, vol. 182, no. 1, pp. 3-12, 2006. 